\title{
Hippocampus detection and calculation of its characteristics in magnetic resonance imaging of the brain
}

\author{
V N Gridin ${ }^{1}$, M I Truphanov ${ }^{1}$ and V I Solodovnikov ${ }^{1}$ \\ ${ }^{1}$ Center of Information Technologies in Engineering RAS, Marshal Biryuzov Str. 7a, \\ Odintsovo, Moscow region, Russia, 143000
}

\begin{abstract}
Hippocampus is the most informative object of the brain for the purpose of detection of Alzheimer's disease signs. This paper presents the approach which was developed for detection of hippocampus and subsequent calculation of its parameters while analyzing the series of images obtained in a sagittal projection by means of a magnetic resonance tomography. This paper introduces the algorithms for detecting the keyframes in the entire series which contain the hippocampus and for identifying the hippocampus among other brain structures. The problems of measuring the volumatric parameters of the hippocampus and calculating its characteristics are considered. These characteristics serve as the basis for instrumental calculation of the signs which characterize the possible presence of Alzheimer's disease.
\end{abstract}

\section{Introduction}

The increased life expectancy of the world's population results in the increase of the occurrence and prevalence of Alzheimer's disease. According to the World Health Organization, the number of people suffering from Alzheimer's disease will double every twenty years and will reach 115.4 million by 2050. Regarding this issue, it is critical to solve the task of identifying the disease at the earliest stage before the cognitive impairments affect the daily activity of a person, while there is still the potential possibility to slow down the progression of thedisease.

Unfortunately, there is no harmless, inexpensive, and most importantly, non-invasive methodto diagnose Alzheimer's disease with a high degree of certainty. Currently,confirmation of the final diagnosis includes a histopathological analysis of brain tissue or the cerebrospinal fluid study in order to determine the formation of beta-amyloid, the characteristic of Alzheimer's disease, for which the patient is required to undergo a spinal cord puncture. Also, there is a method of positron emission tomography of amyloid based on the use of radioactive material, which is a technologically complex and expensive process and implies the effect of radioactive radiation on the examined person. In this case, particular importance is acquired by the tasks related to development of the automated methods, algorithms, software and hardware to detect abnormalities in the structure and activity of the brain which are based on the visual analysis of optical cross-sections of the head in various planes obtained with the help of a magnetic resonance tomography (MRI).

The extensive range of works is devoted to the problems of the construction of the automated system for MRI images processing aimed to analyze the morphological features, which are inherent to Alzheimer's disease. Most of these works note that, first of all, the changes affect the temporal region of the brain and especially hippocampus. Thus, the primary and essential tasks are the detection of 
hippocampus and calculation of its volumetric parameters for the purpose of the automated diagnosis of Alzheimer's disease [1,2].

Currently, various software products (both commercial and freeware) are known that are used to automate the measurement process and calculate the characteristics of brain structures, in particular, the hippocampus and adjacent areas [3,4]. Their common drawback is the mandatory use of some manual operations which complicates the analysis process and leads to the errors in measurement of the key characteristics. The consequence is the potential errors in the diagnosis.

Thus, in [5] area measurements of the hippocampal region of rats brain were performed by means of analysis of the images with the help of a specialized software tool, focusing on the "Rat Brain" atlas, Paxinos G. and Watson C. The authors have used this atlas to find pre-known positions of the hippocampus in the general structure the brain. Obviously, an approach based on the use of atlas can be applied only for reading the estimated coordinates of the hippocampal region. It requires considerable refinement for the purpose of detection and measurement of the linear and volumetric parameters of the hippocampus.

In [6], the following digital image processing operations were applied providing the solution of a part of the problem in automatic mode: subtraction of the background to minimize the influence of the background component; the transformation of the image into monochrome; increasing of contrast; binarization; noise suppression. However, it is indicated that these operations were performed in manual mode using specialized software. The results of using this approach present a certain interest in terms of determining the certain parameters of individual steps during automated visual data processing.

The key features of the hippocampus structure are specified in $[7,8]$. The authors in [8] carried out the research of the hippocampus images using specialized programs in an automated mode in the "FreeSurfer" software environment, as well as in semi-automatic and manual modes in the "Display" package. This software allows a user to perform operations on allocation of the brain structures and their volumetric evaluation. Standard operations of digital image processing (contour highlighting, binarization, etc.) were used to obtain the results. However, human participation was still necessary for the detection of the hippocampus. These approaches are not applicable for the automatic evaluation of the volumetric and dimensional parameters of hippocampus and adjacent brain structures.

In a number of works, the analysis of statistical characteristics and texture analysis as well as artificial neural networks are used to highlight brain structures (including hippocampus) $[9,10,11]$.

In particular, in [12] the authors showed the possibility of using texture image characteristics together with an artificial neural network for analysis of the hippocampus region. However, a large training sample is needed to use this approach, which complicates the practical application of this approach. Another difficulty includes the automatic normalization which is necessity for orientation and spatial characteristics of the three-dimensional regions formed during the analysis of textures, which also reduces the practical value of this approach.

The issue of tomographic images analysis automation for other internal organs also becomes more and more relevant. So in the article [13] the simplest technology of automatic recognition of emphysema of lungs by sets of two-dimensional diagnostic images of computed tomography is considered. In [14], a method for segmentation of organs of the retroperitoneal space on tomographic images based on the level function was proposed.

In this connection, it can be concluded that further research is needed to improve the methodology and mathematical apparatus for automation of magnetic resonance imaging analysis and the calculation of hippocampus characteristics as a key informative brain structure, which would be an essential step in the Alzheimer's disease diagnosis.

\section{The key stages in processing the series of MRI images}

The developed approach for the visual MRI data processing aims to create tools for constructing automatic subsystems of analysis of brain structures images, in particular, the hippocampus for the Alzheimer's disease diagnosis. The following main stages could be picked out:

- selection of series slices in the sagittal projection, presumably containing the hippocampus; 
- subsequent iterative processingin order to confirm /not confirm the detection of the hippocampus in each frame;

- clarifying the location of the hippocampus and calculating its characteristics by analyzing a series of neighboring frames or by repeating the selection of frames sequence presumably containing the hippocampus;

- transmission of the received parameter vector to the decision support module to get information about the possible presence of the Alzheimer's disease, the healthy state or the presence of brain changes not caused by Alzheimer's disease.

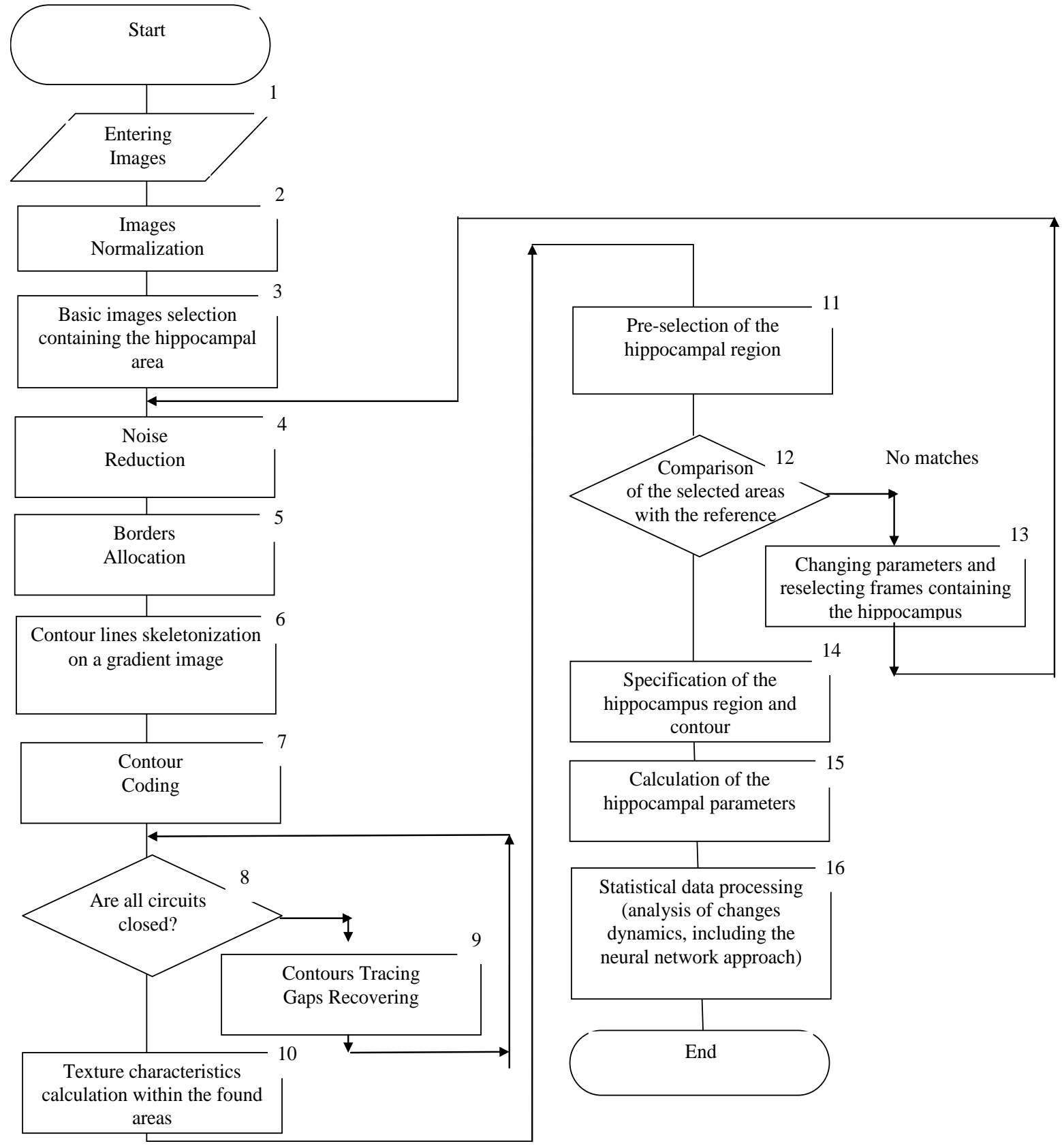

Figure 1. A generalized algorithm for hippocampus detecting and measuring of its parameters for the Alzheimer's disease signs detection.

Let's consider the first group of operations aimed at the slice search and selection in the MRI sequence containing the hippocampus. Note that here and further we consider the location of the hippocampus in the left and right hemispheres of the brain. The main idea is to sequentially scan 
images in the saggital projection and search for a structure that is supposedly appropriate to the hippocampus shape and location. The location is clarified in relation to the location of the eye, which is very informative since it is characterized by a unique close to round shape and as having a closed contour. Also, the calculations of the brain eccentricity and the neck vessels eccentricity are used to determine the orientation in space and the boundaries of the brain as the closed objects which have the largest area. The brain boundaries are calculated by detecting several curves of identical shape and length, located close to each other. These curves are the boundaries of the cranium and intracranial fluid.

The generalized algorithm of the proposed approach is shown in Figure 1.

\section{The key stages for the series of MRI images processing}

The hippocampus could be detected in a series of consecutive frames, on which there is an elongated body framed by a liquid, characterized by a closed contour of a pre-known shape and with precomputed textural characteristics.

Figure 2 presents images, which illustrate the process of primary detection of the hippocampus in a series of sagittal MRI images.
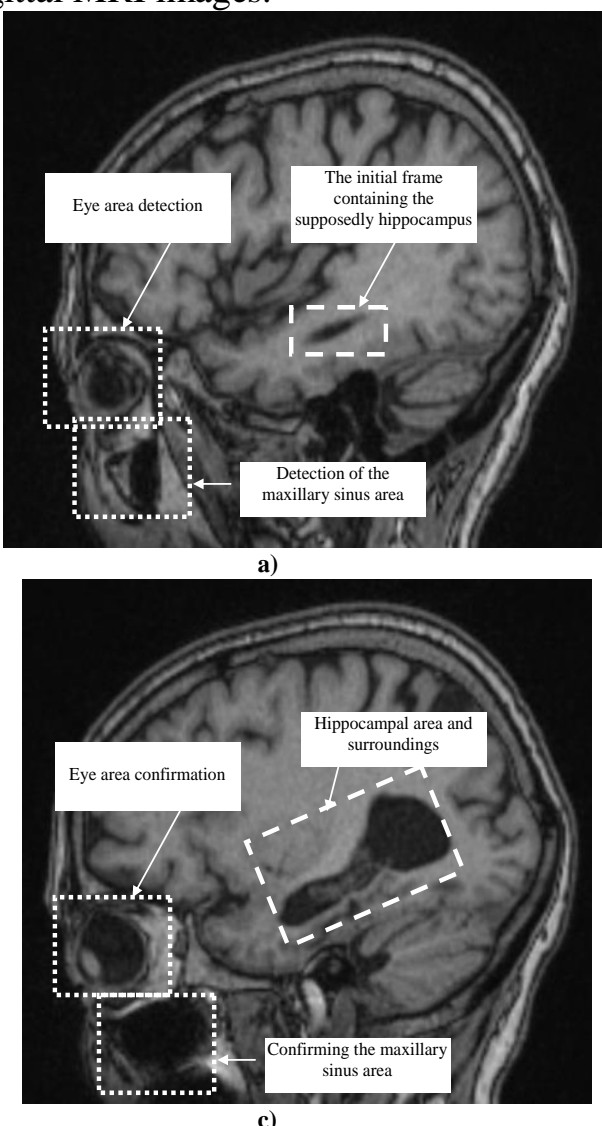

c)

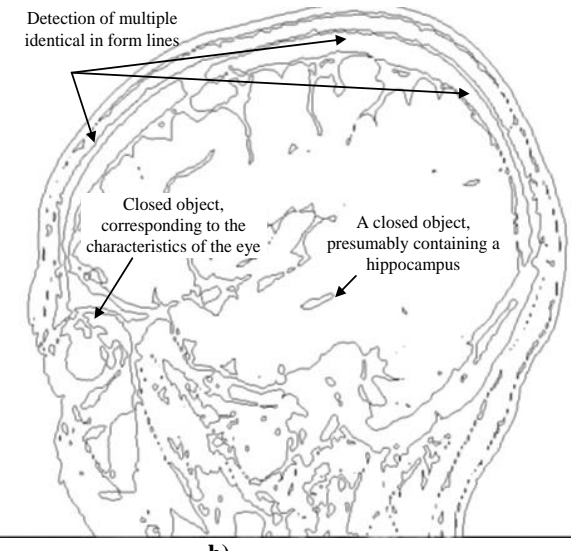

b)

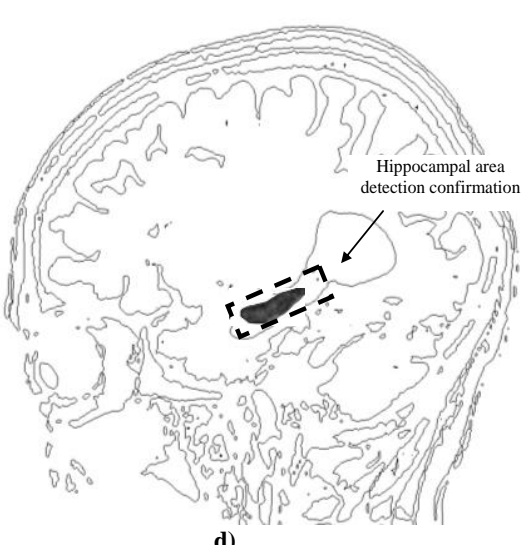

d)

Figure 2. The process of search of the hippocampus: a, b) - the initial frames presumably containing the hippocampus; $\mathrm{c}, \mathrm{d}$ ) the confirmed frames containing the hippocampus in the middle of the sequence.

Thus, the search in the general sequence of sagittal images is directly related to the images detection on which the eyes and maxillary sinuses could be detected, as the most informative and precisely localized objects. Then, the skull boundary and the brain boundary are determined on the found sections to establish the relative position of the hippocampus and to calculate its potential location. Next, by analyzing a series of successive frames, it is iteratively determined those frames on which there is a region corresponding to a generalized description of the brightness and spatial characteristics of the hippocampus. The confirmation of the detection is made at the final stage by 
constructing a closed contour for boundaries description. In addition, the comparison with the reference values and parameters of the adjacent hippocampus regions takes place.

If the hippocampus detection was not confirmed, the initial frame of the sequence is selected again and the search process has to be repeated with the changed parameter values.

\section{Determination of the hippocampus boundaries and position}

Let's describe the process of precise determination of the hippocampus boundaries and position in more details. This process consists of the following main steps:

- pre-processing - reducing the random noise level by a Gaussian filter and adjusting the contrast to the reference parameters [15];

- locating the objects boundaries in the image by means of a differential operator;

- binarizationof the obtained boundaries by the threshold operator with adaptive threshold calculation based on the Otsu method;

- skeletonizationof the obtained contours and restoration of discontinuities in contours with the help of the initial halftone image analysis;

- additional confirmation of the detected hippocampus on the basis of generalized spectral characteristics of local image areas;

- inspecting the series of frames to determine the size of the hippocampal regions relative to the total brain size, and calculating the dimensions of the fluid, which adjacent to the hippocampus.

The key steps of processing each MRI image frame are shown in Figure 3.

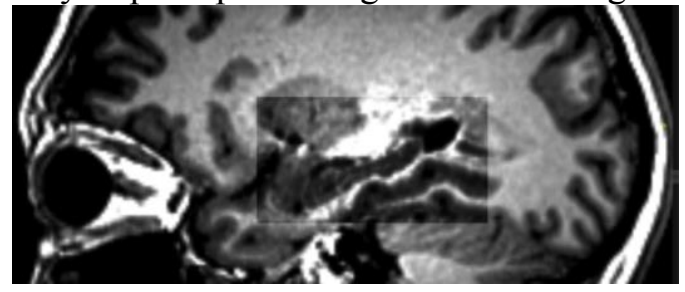

a) - contrast change

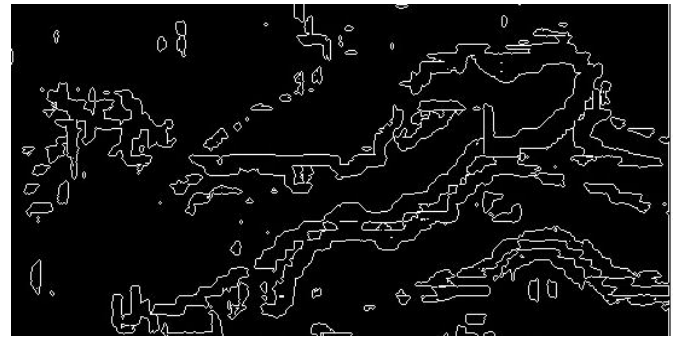

c) - contours binarization and skeletonization

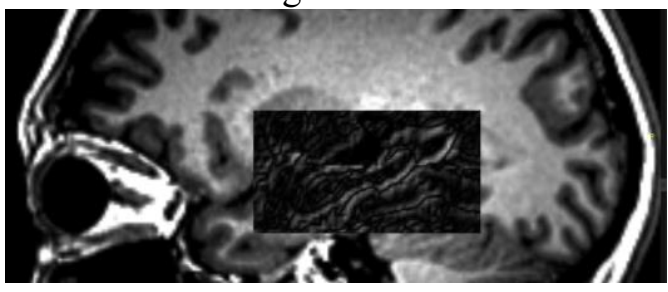

b) - image area differentiation

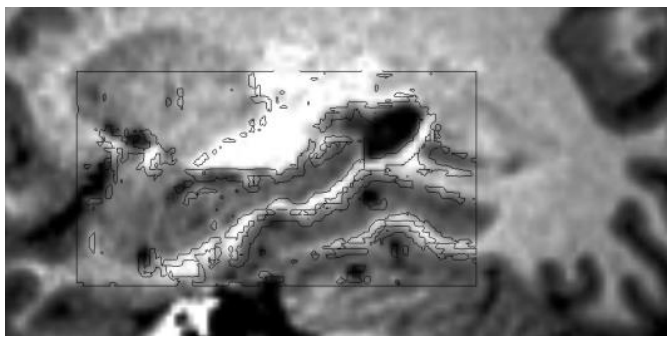

d) - spectral parameters calculation

Figure 3. The process of the hippocampus detection confirmation and its characteristics determination.

\section{Noise elimination}

Noise elimination consists of smoothing and eliminating of sudden jumps of brightness associated with the physical process of magnetic resonance imaging and the intrinsic noise of the tract of tomograph.

The filtering process is based on the calculation of the new luminance value $g$ with a help of the Gaussian filter for each discrete image point according to the formula:

$$
\mathrm{g}(\mathrm{x}, \mathrm{y})=\frac{1}{\sqrt{2 \pi} \sigma} \mathrm{e}^{-\frac{\mathrm{d}^{2}}{2 \sigma^{2}}},
$$

where $\sigma$ is the filter parameter, $d=\sqrt{\left(x-x_{c}\right)^{2}+\left(y-y_{c}\right)^{2}}$ is the distance of the $[x, y]$ pixel from the center pixel of the neighborhood $\left[\mathrm{x}_{\mathrm{c}}, \mathrm{y}_{\mathrm{c}}\right]$, which determines the radius of the filter. 


\section{Selection of contours}

The gradient vector of the image brightness function is calculated to extract the contours at each point. This gradient vector is described by the module $g(x, y)$ and the direction. The set of gradient vectors at each point of the filtered image $\mathrm{f}^{\prime}(\mathrm{x}, \mathrm{y})$ is a gradient image [16]

$$
G r=\langle g(x, y), v(x, y)\rangle, x=\overline{1, K}, y=\overline{1, N},
$$

containing information about the differences in the brightness function, i.e. about contour lines $(\mathrm{K}, \mathrm{N}$ are the image dimensions).

Calculation of the hippocampus and adjacent areas parameters is performed on a series of adjacent frames and the contour image shown in Figure 4.

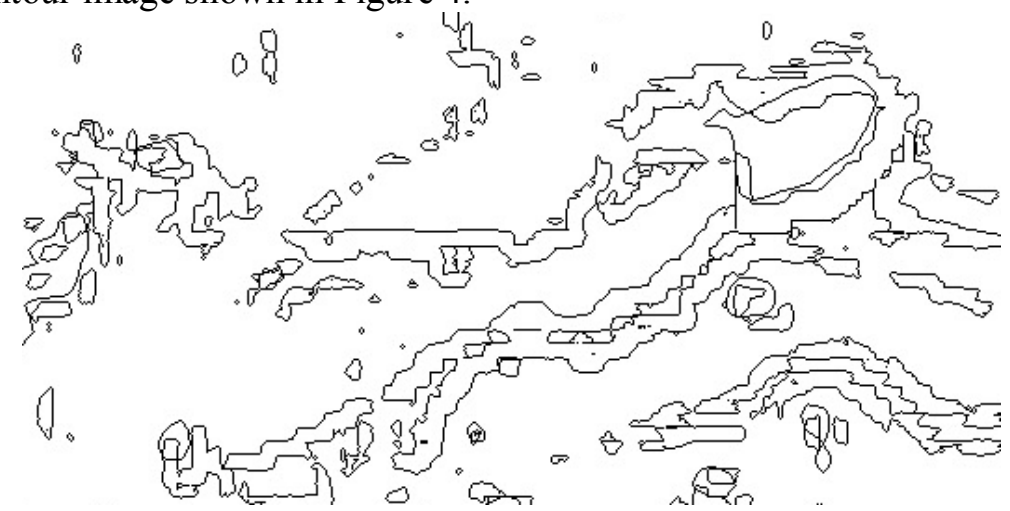

Figure 4. Contour image which is used to calculate hippocampal parameters.

It is worth noting that the obtained absolute values characterizing the state of the hippocampus, in the future, have to be transformed into relative values taking into account the total volume of the brain and the patient's gray matter. Also, all changes in the form of the hippocampus itself and of the surrounding areas could play an important role in the diagnosis. These changes, in turn, could be revealed by the resulting contour images.

\section{Conclusion}

The analysis of scientific, patent sources and reference information revealed that there is a lack of a developed algorithmic and software-hardware solution for automatic analysis of MRI data in order to detect abnormalities in the structure and activity of the brain inherent to Alzheimer's disease at an early stage. The task of the automatically hippocampus detection on MRI images and selection of the most informative images in a general sequence is the key task while constructing both automatic and automated means of calculating signs, which characteristic the Alzheimer's disease. This paper suggests an approach for automatic detection and measurement of the spatial characteristics of the hippocampus, as well as the allocation of nearby areas. This information is used as a characteristic space for making a decision about the possible presence of the disease. The practical significance of the being developed approach lies in the subsequent construction of a specialized domestic software product that allows automatic and automated analysis of the brain MRI images in the interests of timely detection of Alzheimer's disease and instrumental evaluation of its progress dynamics.

\section{References}

[1] Yahno N N, Zaharov V V and Lokshina A B 2011 Dementia: hand-in for doctors (Moscow: MED press-inform) $\mathrm{p} 272$

[2] Lobzin V Yu, Kiselyov V N, Fokin V A, Emelin A Yu, Vorobyov S V, Lupanov I A, Sokolov A V and Efimcev A Yu 2013 Magnetic resonance morphometry usage in the diagnosis of Alzheimer's disease and vascular cognitive disorders Bulletin of the Russian Military Medical Academy 3(43) 1-7

[3] Magonov E P, Kataeva G V and Trofimova T N 2015 Modern methods of automatic calculation of intracranial space volume with MRI-morphometry of the brain Journal of the Bulletin of 
Novgorod State University. Yaroslav the Wise 2(85) 98-104

[4] Chow N, Hwang K, Hurtz S, Green A, Somme J, Thompson P, Elashoff D, Jack C, Weiner M and Apostolova L 2015 Alzheimer's Disease Neuroimaging Initiative. Comparing 3T and 1.5T MRI for mapping hippocampal atrophy in the Alzheimer's Disease Neuroimaging Initiative AJNR Am J Neuroradiology 36(4) 653-660

[5] Sidorova M V and Vakolyuk I A 2017 Determination of the hippocampus structural changes in the early period of modeling of post-traumatic stress disorder in rats International Journal of Applied and Fundamental Research 7(1) 106-110

[6] Tishkina A O 2009 The automatic quantitative analysis method of brain sections microphotographs Neurochemistry 26(4) 341-346

[7] McLean J 2012 The investigation of hippocampal and hippocampal subfield volumetry, morphology and metabolites using $3 T$ MRI (Thesis for the degree of Ph.D. University Glasgow) p 354

[8] Ananeva N I, Ezhova R V, Galsman I E, Davlethanova M A, Rostovceva T M, Stulov I K, Vasserman L I, Shmeleva L M, Chujkova A V, Sorokina A V and Ivanov M V 2015 Hippocampus: radial anatomy, structural variants Radiation diagnostics and therapy 1(6) 39-44

[9] Patil M M and Yardi A R 2011 Diagnosis of Alzheimer's Disease from 3D MR Images with Statistical Features of Hippocampus CIIT, CCIS 250 744-749

[10] Kodama N, Kawase Y and Okamoto K 2016 Application of Texture Analysis to Differentiation of Dementia with Lewy Bodies from Alzheimer's Disease on Magnetic Resonance Images IFMBE Proceedings 14(3) 1444-1446

[11] Rabeh A, Benzarti F and Amiri H 2016 Diagnosis of Alzheimer Disease from MRI Images of the Brain Throughout Time Proceedings of the 16th International Conference on Hybrid Intelligent Systems, Advances in Intelligent Systems and Computing 552 219-226 DOI: 10.1007/978-3-319-52941-7 22

[12] Jing Z, Yu C, Jiang G, Liu W and Tong L 2012 3D texture analysis on MRI images of Alzheimer's disease Brain Imaging and Behavior 61-69

[13] Smelkina N A, Kolsanov A V, Chaplygin S S, Zelter P M and Khramov A G 2017 Pulmonary Emphysema Recognition by CT Scan Computer Optics 41(5) 726-731 DOI: $10.18287 / 2412$ 6179-2017-41-5-726-731

[14] Eruslanov R V, Orehova M N and Dubrovin V N 2015 Retroperitoneal Space Organ Segmentation From CT Images Based on the Level Set Function Computer Optics 39(4) 592599 DOI: 10.18287/0134-2452-2015-39-4-592-599

[15] Gridin V N, Titov V S and Trufanov M I 2009 Adaptive vision systems (SPb.: Science) p 441

[16] Furman Ya A 2002 Introduction to contour analysis and its applications to signal and image processing (Moscow: Fizmatlit) p 592

\section{Acknowledgments}

The study was carried out at the expense of a grant from the Russian Science Foundation (project 1711-01288). 